\title{
Erratum: Ultrafast Spin-State Dynamics in Transition-Metal Complexes Triggered by Soft-X-Ray Light [Phys. Rev. Lett. 118, 023001 (2017)]
}

\author{
Huihui Wang, Sergey I. Bokarev, Saadullah G. Aziz, and Oliver Kühn
}

(Received 5 June 2018; published 28 June 2018)

DOI: 10.1103/PhysRevLett.120.269901

The values of the pulse durations and respective bandwidths in the text and figure captions of the original Letter do not correspond to the actual pulse envelopes shown in the population graphs or those used to trigger dynamics in the numerical simulations. The correct temporal durations (full width at half maximum, FWHM) should read $\sigma=0.132$ and $1.32 \mathrm{fs}$, and the respective bandwidths are $h / \sigma=31.4$ and $3.1 \mathrm{eV}$. The numbered ranges in panel (b) of Fig. 1 of the original Letter also need to be corrected as shown in the figure below. For convenience, we also denoted the carrier frequencies of the respective pulses with dashed lines.

These changes alter neither the results shown nor the conclusions drawn in the Letter.

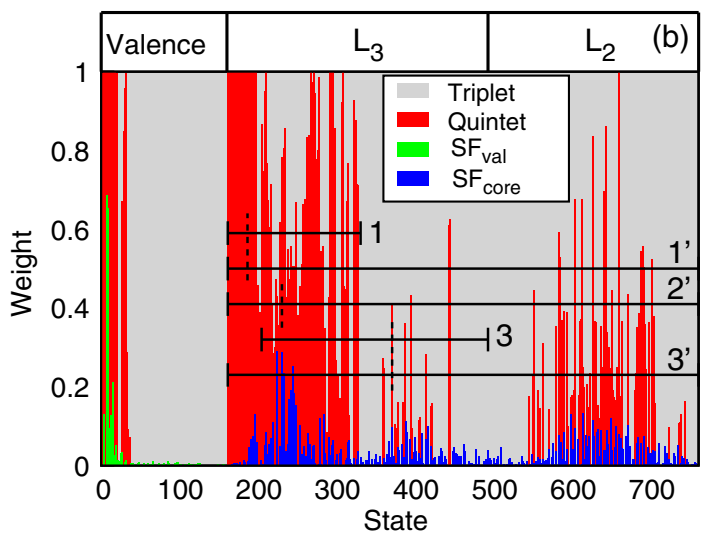

FIG. 1. [Corrected Fig. 1(b)] Collective contributions of the quintet (red bars) spin-free (SF) states to the stationary spin-orbit coupled (SOC) eigenstates $\sum_{j, M_{S}}\left|c_{j}^{\left(S=2, M_{S}\right)}\right|^{2}$, see Eq. (1). The grey areas correspond to the triplet SF states $\left(\sum_{j, M_{S}}\left|c_{j}^{\left(S=1, M_{S}\right)}\right|^{2}\right)$, which together with the quintet ones, sum up to unity. The particular contributions of valence $\mathrm{SF}$ states $\left(\mathrm{SF}_{\mathrm{val}}\right.$, green bars) and core $\mathrm{SF}$ states $\left(\mathrm{SF}_{\mathrm{core}}\right.$ blue bars) to the different SOC states are also shown. Numbered horizontal bars reflect the bandwidths of 3.1 eV (1 and 3) and $31.4 \mathrm{eV}$ (1', 2', and 3') pulses with carrier frequencies (marked by dashed lines) as given in panel (a) of Fig. 1. 